\title{
Novel and Efficient Synthetic Microvascular Anastomosis Training Model
}

\author{
Yun-Huan Hsieh, MBBS, MS1,2; Tommy Nai-Jen Chang, MD*
}

'Department of Plastic and Reconstructive Surgery, Chang Gung Memorial Hospital, Linkou Medical Center and Chang Gung Medical College and Chang Gung University, Taoyuan, Taiwan

${ }^{2}$ Center for Vascularized Composite Allotransplantation, Department of Plastic and Reconstructive Surgery, Chang Gung Memorial Hospital, Chang Gung Medical College, and Chang Gung University, Taoyuan, Taiwan

\begin{abstract}
Microsurgery is an essential surgical skill of modern Plastic and Reconstructive Surgery, and it has now well integrated into the practice of many other surgical specialties. With the growing demand of this surgical skill, having a cost-effective training model that is globally accessible is indispensable. This model was made from widely available non-sterile latex gloves with tools and materials that are readily accessible in the laboratory. Similar to other existing latex models, this model is economical and delivers good simulation of the arterial wall elasticity and thickness. It is a suitable model for group training sessions due to its simple construct, where a layperson can be employed for the model setup. Based on our current experience in the training courses, this is a useful bridging model between basic microsurgical exercises and advance microsurgical training with in vitro or in vivo animal models. This non-commercialized model provides a simple platform for trainees to perfect their microanastomosis techniques ethically, systematically, and cost-effectively.
\end{abstract}

\section{Introduction}

Microsurgery creates a new frontier in modern surgical practice. Its application has broadened beyond the realm of Plastic and Reconstructive Surgery, and now integrated as part of standard practice in many surgical specialties [1]. From free flap reconstructions to allotransplantation of hands and faces, the development and advancement of microsurgery realizes the grand visions of surgical giants from generations before. Similar to musician training, vigorous and dedicated microsurgery exercise is the only path for its technical perfection. Abundant training models are developed and described in the literature since the birth of microsurgery in 1960s [2]. In vivo animal model is ideal for pre-clinical training, where every facet of microsurgery techniques can be exercised with higher complexity, and the end result of the anastomoses can be observed directly. However, it is a complex model for a novice, and the excess use of live animals for surgical training also raised ethical, economical, and logistic conflicts [3-7]. Many non-animal organic and synthetic models are adopted by microsurgical training centers as their first line training methodology. These models, unlike the in vivo animal models, can be tailored to facilitate targeted exercises, thus trainees can build their technical skills ethically, cost effectively, and systematically $[4,5,7]$. Among them, latex based models are the most common and has been used for microsurgical training for more than 3 decades [8-13]. The complexity of the model construct varies from 2D flat sheet exercises $[8,9]$ to $3 \mathrm{D}$ tubule anastomoses [10-14]. Latex not only simulates arterial lumen well but is also affordable and widely available. This article is aimed to present a novel model based on 3D latex vessel construct for microvascular anastomosis training and compare it with other latex based training models described in the literature.

\section{Method, Material, and Training Model}

The lead author, Dr. Yun-Huan Hsieh, invented the model based on the available resources in Vascularized Composite Allotransplantation Center, Chang Gung Memorial Hospital, Taiwan. Resources required for this exercise set up include a pair of scissors, a latex glove, a rat board with $1 \mathrm{~cm}$ hollow cylindrical attachment, Micropore Paper Medical Tape (Micropore, 3M), 10-0 nylon (ETHILON ${ }^{\mathrm{TM}}$ 10-0 Nylon Suture), microsurgical instruments, and a table microscope (Carl Zeiss, Germany). The latex glove is transected at the tip and the phalangeal base (Figure 1), creating a finger latex tube. Two strips of Micropore tapes were applied over the mid portion of the finger latex tube, one over the dorsal surface and the other over the ventral surface. The tapes extend and seal over the edge of the latex tube both proximally and distally while the latex tube is in slight extension. Once the inner space is obliterated by the sandwiching technique with the tapes, two tubular structures, the synthetic vessels, are effectively constructed (Figure 2). Its vessel dimension (size) equals to the distance from the edges of the tapes to edges of the finger latex tube (Figure 3). The finger latex tube is then suspended and fixed over the hollow cylindrical attachment of the rat board with Micropore Tapes (Figure 4). The constructed system is positioned under the microscope, with magnification ranges between 20x to 40x. Double microvascular clamps is placed over one of the synthetic vessels, microvascular anastomosis with 10-nylon can begin once the vessel is transected (Figure 5).

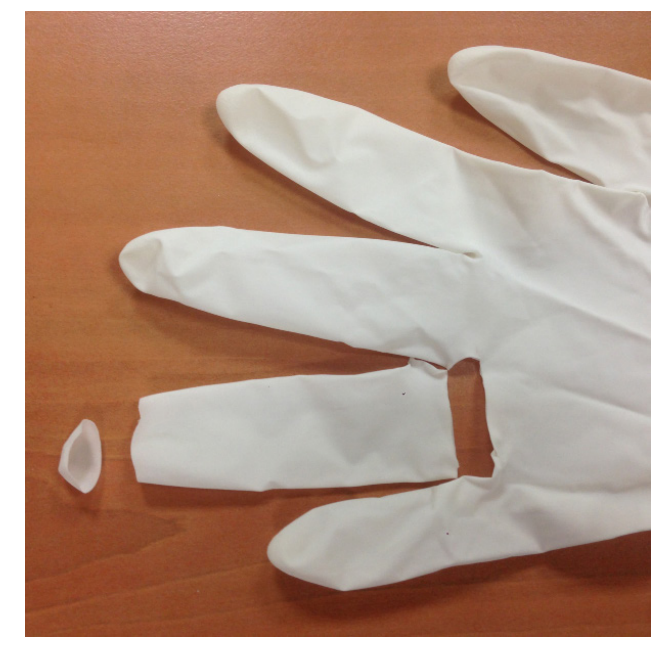

Figure 1. The latex glove is transected at the tip and the phalangeal base, creating a finger latex tube. 


\section{IDEA AND INNOVATION}

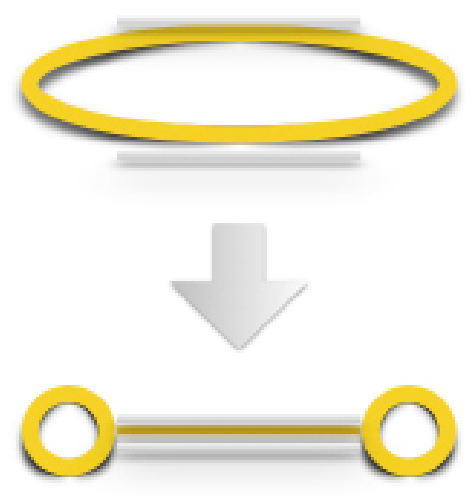

Finger Latex Tube before sandwich taping with Micropore Tape

\section{Finger Latex Tube after sandwich taping with Micropore Tape}

\section{Finger Latex Tube}

Figure 2. Two strips of Micropore tapes were applied over the mid portion of the finger latex tube, one over the dorsal surface and the other over the ventral surface. The tapes extend and seal over the edge of the latex tube both proximally and distally while the latex tube is in slight extension. Synthetic vessels (x2), is thus created as the inner space of the finger latex tube is obliterated by the sandwiching technique with Micropore tape.

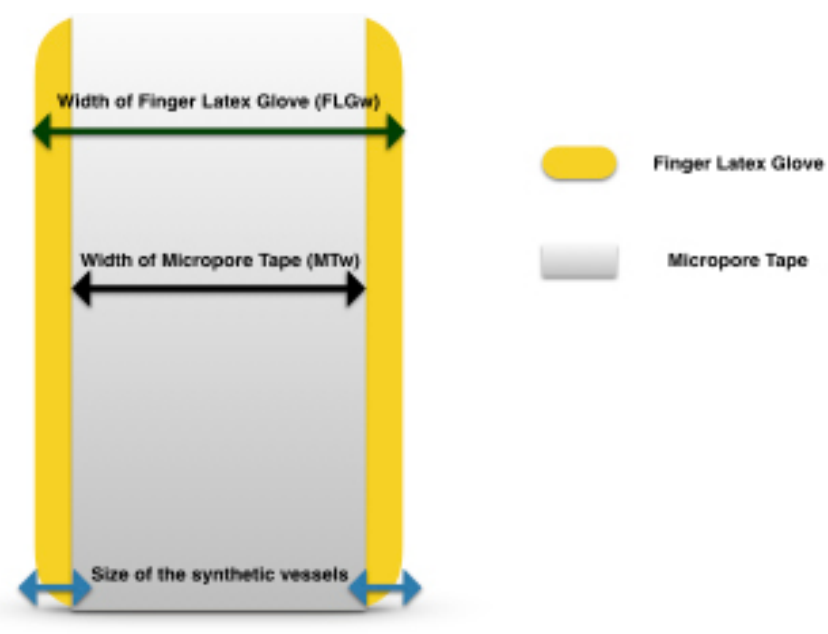

\section{Synthetic vessel size $=1 / 2(F L W w-M T w)$}

\section{Distance between the edge of the finger latex glove and micropore tape}

Figure 3. The dimension (size) of the synthetic vessels equals to the distance from the edges of the tapes to edges of the finger latex tube.

\section{Utilization and Experience of Novel Synthetic Microanasto- mosis Model}

Taiwan Society of Reconstructive Microsurgery (TSRM) adopted this model for their Microsurgery Training Courses since 2016. It was a 4-day course, providing basic proficient microsurgical skills for the trainees. In addition to lecturing the basic concepts of microsurgery, trainees were presented with series of tasks including knot-tying exercise between threads of a gauze with 10-0 nylon, simple interrupted suture practice on a suspended flat latex sheet, synthetic vessel microanastomoses (this model), and chicken wing brachial vessel anastomoses. This model required intermediate level of micro-instrument handling as well as proficient suturing techniques. Therefore, it was used as a bridging exercise prior to the actual vascular anastomoses. Although not subjectively measured nor compared, this synthetic microanastomoses model was a beneficial bridging exercise reported by most trainees in the course.

\section{Discussion}

Perfection in microvascular anastomosis technique requires devoted training to overcome its hurdles and challenges. The live rat remains to be the gold standard microsurgical training model [1,15-17], it offers adjunctive practice on tissue dissection, adventitial striping, as well as enhancing 3D perception and enabling immediate vessel flow test in addition to microvascular anastomoses exercise [17]. The rising concerns of laboratory animal welfare promotes ingenious ideas into novel training model innovations. Bubble wraps [18], parafilm [19], foliage leaves [20], and gauze $[21,22]$ are commonly used for fundamental microsurgery training. Commercially available training cards with silicon tubes [22], synthetic vessels with polyurethane [3], polytetrafluoroethylene [23], and polyethylene $[24,25]$ are useful models for advanced microsurgical training in attempt to reduce animal usage. Similarly, cryogenic rat or rabbit femoral vessels, carotid vessels, and aorta offer "off the shelf" microvascular anastomosis exercise from the "recycled parts" of the sacrificed animals [26]. These or- 


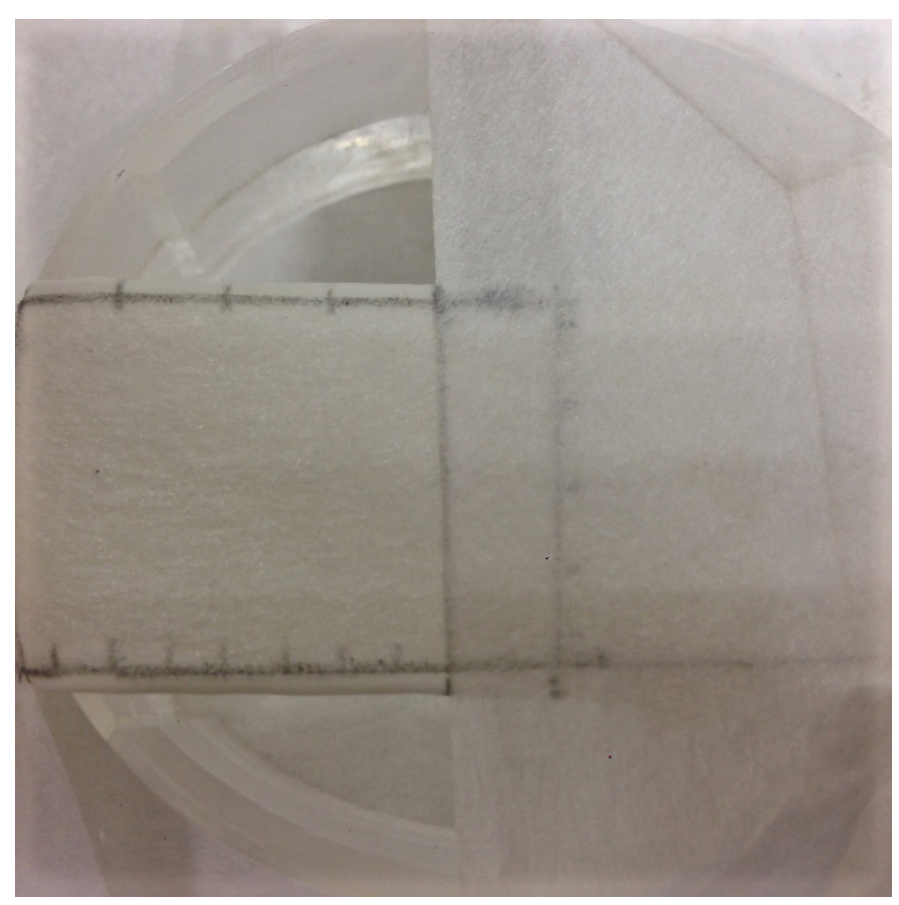

Figure 4. The finger latex tube is suspended and fixed over the hollow cylindrical attachment of the rat board with Micropore Tapes.

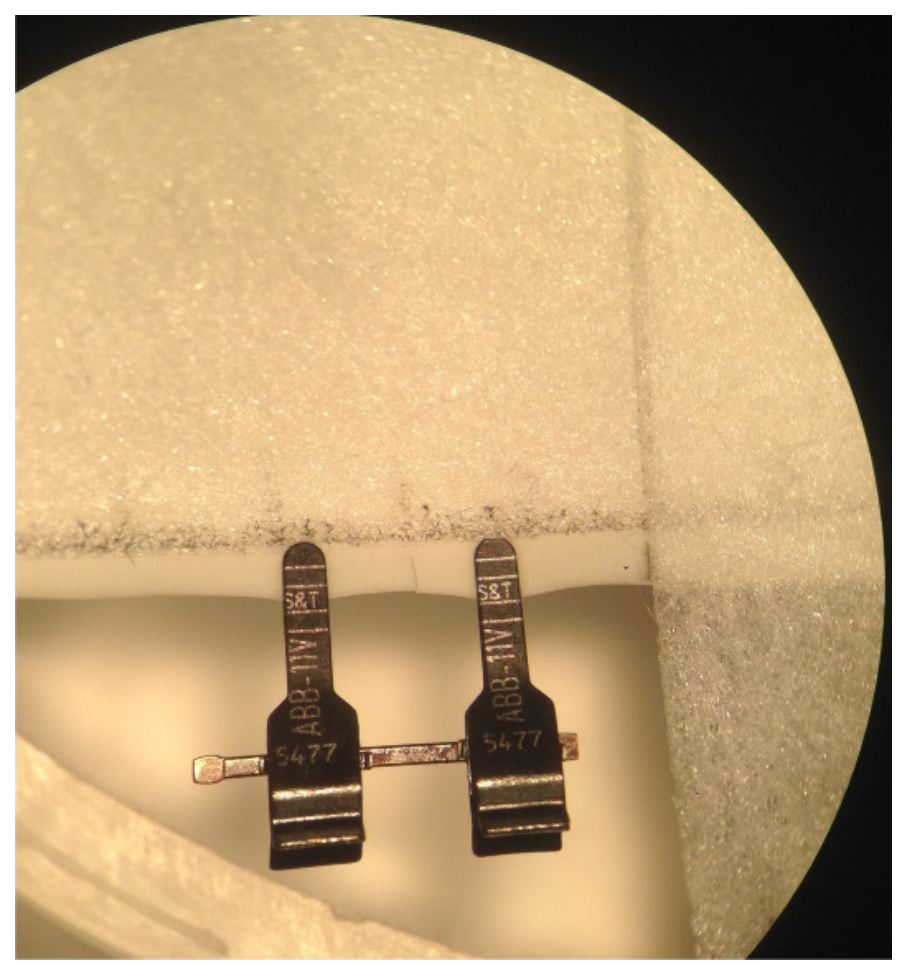

Figure 5. Microvascular anastomosis with 10-nylon can begin once the vessel is transected, and a double clamp is applied.

ganic and synthetic vessels provide good targeted simulations for vessel anastomoses but unable to offer practice of abovementioned adjunctive exercises. Due to commercialization of the synthetic vessels, and labor intense preparation of the cryogenic vessels, these models are less accessible and are less cost effective to many training centers.

Latex based microvascular anastomosis models have been utilized for more than 3 decades [8-13]. General advantages of using latex for the

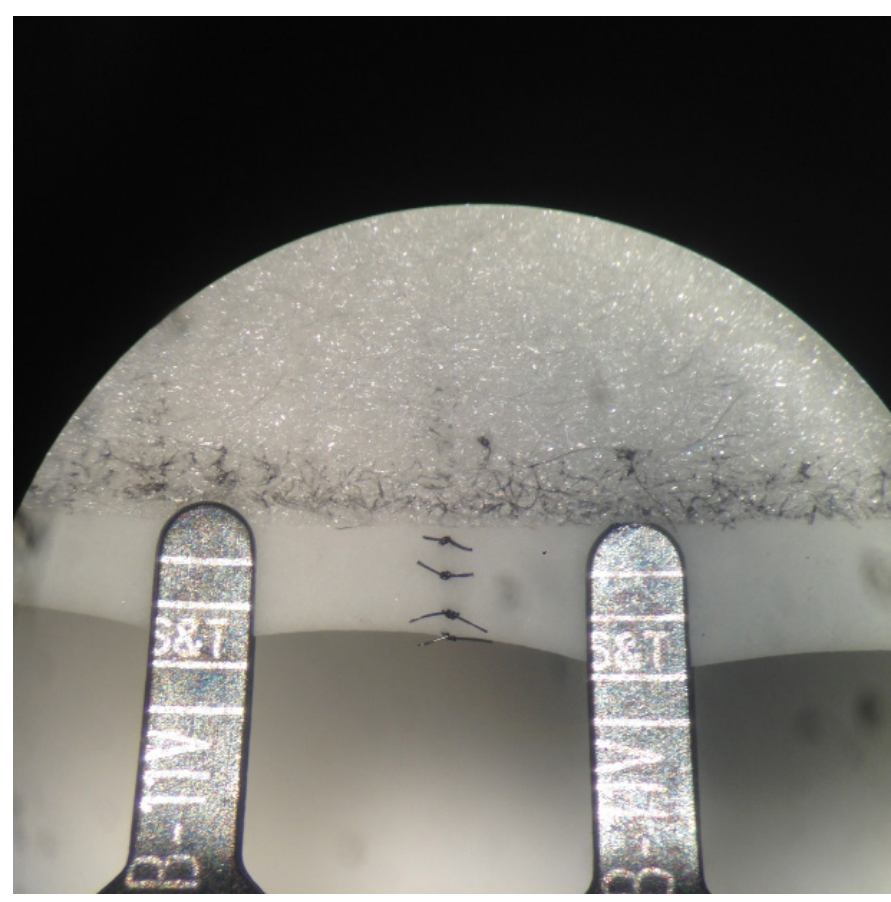

Figure 6. The completion of anterior wall anastomosis.

construct of microvascular anastomosis models include: (1) availability and accessibility of material in all surgical centers and laboratories, (2) economic benefits, and (3) simulation of elasticity and thickness of the arterial wall. The complexity of the described models varies; Awwad [7] and Lee [8] described 2D models for basic microsurgical exercises, comprising basic micro-instrument handling, suture spacing, needle passage, and knot tying in different angles. More complex models are constructed in 3D, resembling authentic "tubule vessels" for microanastomosis training. Guler and Rao [15] described a simple method of tubularization from a latex sheet. They used the cuff of a latex glove, secured over the open ends of a suture box. 2 parallel $10 \mathrm{~mm}$ slits were made, creating a central latex strip. Suturing free edges of the latex strips with 10-0 nylon to construct the prefabricated latex tube. This model was further modified by Crosby et al. [16], by creating 2 latex tubes, allowing end to side, side to side anastomosis as well as vein graft practice in addition to end-to-end anastomosis. Kamath [18] demonstrated a method of self-manufacturing 3D latex conduits by dipping a $1 \mathrm{~mm}$ K-wire into liquid latex solution, producing a latex tube with $1 \mathrm{~mm}$ diameter. Shakeel et al. [19] shares similar passion in utilizing latex conduits for microsurgical training, instead of prefabricating a latex sheet or self-manufacturing latex conduit from its liquid form, they simply use the rim or the sleeve part of a non-sterile glove, employing its hollow rim construct as their model of latex tube. It is a ingenious modification of a latex glove for anastomosis exercise, however, not every glove comprises a hollow rim at its base, thus the model availability is restricted by the brand of the gloves that is used regionally.

In this model, it shares all the advantages as other latex based models. The set up is relatively simple compared with other latex based 3D tubular models. It takes approximately 5 minutes to set up, using widely available tools and materials in a microsurgical lab or an operating theater. As a non-commercialized training model that is simple to construct with materials that are readily accessible, this model can easily be replicated globally. One non-sterile glove consists of 5 finger latex tubes, which equals 5 practice models with total of 10 synthetic vessels. If assuming one surgical glove costs approximately 1 US dollar, the model is estimated to be 20 cents each. Multiple anastomoses can be performed on each synthetic vessel, once the anastomosis is completed, transecting the nearby segment allows direct observation of anastomosis quality, ensuring "vessel inversion" is achieved with each suture. This model does 
not require any previous microsurgical experience for its setup, thus a layperson can facilitate in the model making process in preparation for a large training session. This model, like all other synthetic models, provides a targeted practice. Although it does not offer exercises in vessel dissection, adventitiectomy, and vascular patency test, it simplifies the anastomosis process by neglecting the need to negotiate with the surrounding tissue during microvascular anastomosis. Negotiating with surrounding soft tissue at the site of microanastomosis can be a source of frustration for a novice trainee. Thus this model may facilitate the confidence building for trainees in pursuing further challenges in in vivo models once their microsurgical technique is matured. The "Vessel Size" in this model is defined as the distance between the edge of the tape and the edge of the finger latex tube. Therefore, the vessel size for the anastomosis exercise is adjustable. The closer the tape to the edge of the tube, the smaller the vessel size it is for the exercise. Suspending the model to a cylindrical structure not only provides the anchorage for the exercise, but also facilitates 3D depth perception training under the microscope. Capturing the posterior wall during microvascular anastomosis is a fatal error, as it leads to inevitable anastomosis failure. In this model, a double clamp can be applied as shown in Figure 6, its application not only closely simulates the actual anastomosis but also allows flipping of the vessel to reveal its posterior wall. With direct examination of suture quality and placement, direct viewing of the lumen from the posterior wall ensures the posterior wall is freed from accidental capturing during anterior wall anastomosis (supplementary video). In addition, this exercise can be oriented in different angle, enabling the practice of microvascular anastomosis in different directions to accomplish the training more comprehensively in preparation of unexpected clinical scenarios. The application of this model is limited by lack of separation of the latex vessel from the trunk of the latex finger tube. Thus this model is inapplicable for end-to-side, side-to-side anastomosis and vein graft training.

\section{Conclusion:}

We present a novel synthetic vascular model for microanastomosis training. This model is suitable for trainees with intermediate level of microsurgical skills, and useful as a bridging model between simple suturing exercise and in vivo rat vessel anastomosis during pre-clinical training. It is an easy model to construct, using widely available materials. It simulates arterial anastomosis well, and enables direct observation and feedback of the anastomosis quality. This model is non-commercialized, cost effective, and is readily accessible. This model allows the trainee to grow their microsurgical skills ethically, systematically and confidently.

\section{Article Information}

*Correspondence: Tommy Nai-Jen Chang, MD

Department of Plastic and Reconstructive Surgery, Chang Gung Memorial Hospital, Linkou Medical Center and Chang Gung Medical College and Chang Gung University, Taoyuan, Taiwan. Email: tommynjchang@yahoo.com.tw

Received: Jul. 31, 2017; Accepted: Nov. 20, 2017; Published: Dec. 29, 2017

DOI: 10.24983/scitemed.imj.2017.00048

Copyright (c) 2017 The Author(s). This is an open-access article distributed under the terms of the Creative Commons Attribution 4.0 International License (CC-BY).

\section{Funding: None}

\section{Conflict of Interest Disclosures: None}

\section{Keywords}

Latex; microanastomosis; microvascular anastomosis; synthetic training model; training model.

\section{Reference}

1. Heiner $\mathrm{H}$, Karl P, Tilgner-Peter A. The technic of microvascular anastomoses: the rat as a model. Zeitschrift Fur Experimentelle Chirurgie 1977;10(6):331-337.

2. Noaman $\mathrm{HH}$. Microsurgery in children: history, indications, precautions, and differences from that of adults. Microsurgery 2008;28:83-84.

3. Meier SA, Lang A, Beer GM. Polyurethane vessels for microvascular surgical training to reduce animal use. ALTEX 2004; 21:135-138.

4. Matsumura N, Hayashi N, Hamada $H$, Shibata $T$, Horie $Y$, Endo S. A newly designed training tool for microvascular anastomosis techniques: Microvascular Practice Card. Surgical Neurology 2009;71(5):616-620.

5. Lannon DA, Atkins JA, Butler PE. Non-vital, prosthetic, and virtual reality models of microsurgical training. Microsurgery 2001;21(8):389393.

6. Hong JW, Kim YS, Lee WJ, Hong HJ, Roh TS, Song SY. Evaluation of the efficacy of microsurgical practice through time factor added protocol: microsurgical training using non-vital material. Journal of Craniofacial Surgery 2010;21(3):876-881.

7. Awwad AM. A training card for microsurgery. Microsurgery 1984;5(3):160.

8. Lee S, Coppersmith WJ. A microvascular surgical practice disc for beginners. Microsurgery 1983;4(1):67-69.

9. Guler MM, Rao GS. Canniesburn "ever-ready" model to practice microsurgery. British Journal of Plastic Surgery 1990;43(3):381-382.

10. Crosby NL, Clapson JB, Buncke HJ, Newlin L. Advanced nonDanimal microsurgical exercises. Microsurgery 1995;16(9):655-658.

11. Ballantyne DL, Rei el RS, Harper AD. A systematic learning program for microvascular technique. Plastic and Reconstructive Surgery 1980;65:80-82.

12. Kamath, J. B., and Kamath, S. J. A custom-made latex conduit for simulation of microvascular repair. Plastic and Reconstructive Surgery 2007;119(5):1634-1635.

13. Dustagheer S, Brown AP. Synthetic latex conduits as an aid for microsurgical training. Plastic and Reconstructive Surgery 2008;122(1):321.

14. Zhang F, Chin BT, Ho PR, Costa MA, Lineaweaver WC, Buncke HJ. Rat tail replantation as a training model for microvascular procedures of digit replantation. Microsurgery 1998;18(6):364-367.

15. Bao JY. Rat tail: A useful model for microvascular training. Microsurgery 1995;16(2):122-125.

16. Abla A, Uschold T, Preul MC, Zabramski JM. Comparative use of turkey and chicken wing brachial artery models for microvascular anastomosis training. Journal of Neurosurgery 2011;115(6):1231-1235.

17. Sener S, Menovsky T, Maas AIR. Use of bubble wrap for microsurgical training. Journal of Reconstructive Microsurgery 2013;29(9):635-636.

18. Ramasastry S, Narayanan K, Angel MF. A simple and inexpensive device for microvascular training. Annals of Plastic Surgery 1985; 14(5):462-464.

19. Kaufiman T, Hurwitz DJ, Ballantyne DL. The foliage leaf in microvascular surgery. Microsurgery 1984;5(1):57-58.

20. Indo M, Tsutsumi K, Shin M. The practice of knots untying technique using a 10-0 nylon suture and gauze to cope with technical difficulties of microvascular anastomosis. World Neurosurgery 2011;75(1):87-89.

21. Inoue T, Tsutsumi K, Adachi S, Tanaka S, Saito K, Kunii N. Effectiveness of suturing training with 10-0 nylon under fixed and maximum magnification (x20) using desk type microscope. Surgical Neurology 2006;66(2):183-187.

22. Rayan B, Rayan GM. Microsurgery training card: a practical, economic tool for basic techniques. Journal of Reconstructive Microsurgery 2006;22(4):273-235 


\section{IDEA AND INNOVATION}

23. Korber KE, Kraemer BA. Use of small-caliber polytetrafluoroethylene (Gore-Tex) grafts in microsurgical training. Microsurgery 1989;10(2):113-115.

24. Yen DM, Arroyo R, Berezniak R, Partington MT. New model for microsurgical training and skills maintenance. Microsurgery 1995;16(11):760-762.
25. Weber D, Moser N, Rosslein R. A synthetic model for microsurgical training: a surgical contribution to reduce the number of animal experiments. European Journal of Pediatric Surgery 1997;7:204-206.

26. Kim DC, Hayward PG, Morrison WA. Training model for microvessel anastomosis. Microsurgery 1994;15(11):820-821. 